\title{
RELACIONES CONSTITUTIVAS DE LOS PERFILES TUBULARES DE PARED DELGADA UTILIZADOS EN LA CONSTRUCCION DE MALLAS ESPACIALES DE NUDOS ARTICULADOS
}

\author{
(CONSTITUTIVE RELATIONS OF THIN-WALLED TUBES USED IN THE CONSTRUCTION OF SPACE \\ TRUSSES)
}

José Ramón Atienza, Carlos Miragall y Ramón Irles, Ingenieros de Caminos, Canales y Puertos.

Cátedra de Estructuras Metálicas de la E.T.S. de Ingenieros de Caminos, Canales y Puertos de la Universidad Politécnica de Valencia

RESUMEN

Una adecuada modelación del comportamiento resistente de las mallas espaciales de nudos articulados pasa necesariamente por una descripción correcta del comportamiento previsible de las barras que las integran. Este artículo se ocupa del estudio de la respuesta resistente de dichas barras considerando las no linealidades mecánicas y geométricas que tienen lugar al agotarse sus capacidades de carga por plastificación a tracción o por pandeo a compresión. Aunque el modelo de análisis utilizado es de carácter general, aquí sólo se aplica a barras de sección hueca circular por ser las más comúnmente empleadas en estos sistemas estructurales.

\section{SUMMARY}

A suitable modelling of the structural behaviour of pin-jointed single and double layer grids requires a correct description of the bars behaviour.

This paper deals with the study of the structural response of these bars, taking into account the material and geometrical non-linearities, which arise when they collapse by either plastifying in tension or buckling in compression.

Although the methodology shown can be applied to bars of any cross-section, in this paper only tubular bars will be considered because they are the most commonly used in these structural systems.

\section{INTRODUCCION}

Dos condiciones limites son las que rigen el dimensionamiento de las mallas espaciales: la primera, el estado límite de servicio, se asocia al adecuado funcionamiento de la malla frente a las acciones características; la segunda, el estado límite último, se asocia al agotamiento resistente de la malla frente a las acciones ponderadas.

Es bien sabido que el comṕortamiento resistente de las mallas espaciales en condiciones de servicio es básicamente lineal, de modo que su estudio se puede abordar con cualquiera de los numerosos programas lineales de cálculo matricial de estructuras de barras que se han desarrollado hasta la fecha. También se sabe que el campo de validez del análisis lineal termina cuando la solicitación de la malla es tal que alguna de sus barras se ve solicitada por cargas que exceden su limite de proporcionalidad, o bien cuando los movimientos de los nudos de la malla son lo suficientemente grandes como para invalidar el planteamiento de las ecuaciones de equilibrio en la geometria no deformada.

Consecuentemente, si se desea analizar el comportamiento de la malla hasta su estado limite último, es necesario considerar los efectos no lineales que inciden en su comportamiento.

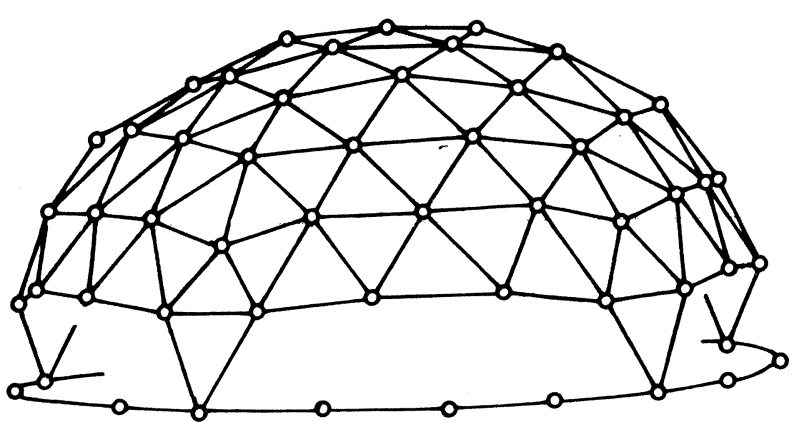

Fig. 1. - Malla espacial de una capa. 
Estos efectos se pueden clasificar en dos grupos: por una parte tenemos las no linealidades geométricas asociadas al movimiento de los nudos de la malla; por otra, las no linealidades mecánicas y geométricas que se desarrollan en las barras de la malla al agotarse sus capacidades portantes por pandeo a compresión o por plastificación a tracción.

El estudio del estado límite último en las mallas de una capa, figura 1, requiere la consideración de todos estos efectos no lineales. En cambio, el mismo estudio en las mallas de varias capas, figura 2, dada su mayor rigidez, sólo suele necesitar la consideración del comportamiento no lineal de sus barras.

El artículo se ocupa de este último tema deduciendo las relaciones constitutivas de los perfiles tubulares comúnmente empleados en la construcción de mallas espaciales de nudos articulados. Dichas relaciones se definen mediante la curva que establece la dependencia entre la carga axil "p" que solicita a la barra y el movimiento relativo "e" de sus extremos medido en la dirección del eje que los une en la geometria deformada.

Conocidas las relaciones constitutivas de las barras biarticuladas que forman una malla espacial, es posible el desarrollo de modelos numéricos sencillos con los que estudiar su estado limite último, tema que dejamos para un próximo artículo.

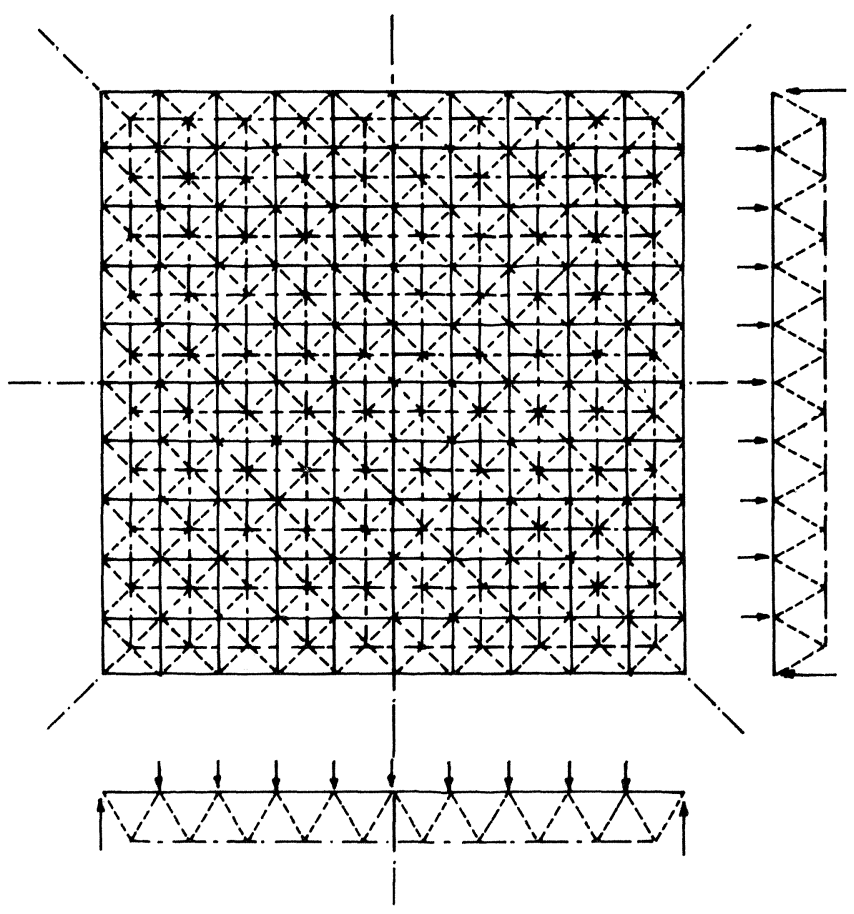

Fig. 2. - Malla espacial de dos capas.

\section{DESCRIPCION DEL COMPORTAMIENTO RESISTENTE DE BARRAS ARTICULADAS SOLICITADAS POR CARGAS AXILES DE TRACCION O COMPRESION}

Existe una marcada diferencia en el comportamiento de las barras "reales" en función de que su solicitación sea de tracción o de compresión. Esta diferencia radica en que las «imperfecciones» de la barra no afectan al valor de su resistencia última a tracción, mientras que, en cambio, sí tienen una influencia decisiva en el valor de su resistencia última a compresión. Así pues, la descripción del comportamiento a tracción de la barra «real» se puede hacer despreciando la influencia de las «imperfecciones", tal como se indica en la parte inferior de la figura 3 , mientras que esta simplificación no es admisible cuando se pretende describir su comportamiento a compresión, representado en la parte superior de la misma figura.

En este punto conviene aclarar que con la palabra "imperfección", siguiendo la denominación usual en los trabajos relativos a la estabilidad de barras comprimidas, hacemos referencia a factores diversos entre los que destacan: la combadura inicial de la barra, la excentricidad inevitable en la aplicación de la carga axil y la no homogeneidad del material estructural ligada básicamente a las tensiones residuales inducidas en los procesos de fabricación (laminación, conformado, oxicorte, soldadura, etc.).

En lo que se refiere al comportamiento a tracción de la barra biarticulada, perfecta o imperfecta, es de destacar que su agotamiento implica que mientras aumenta el alargamiento de la barra se sigue resistiendo la carga de plastificación, e incluso se puede resistir cargas superiores a dicho valor si el alargamiento relativo alcanza la zona de endurecimiento del material por deformación. Por el contrario, el agotamiento a compresión va normalmente acompañado de una pérdida brusca de la capacidad de carga de la barra pandeada a medida que aumenta su acortamiento. Describiendo con más detenimiento el comportamiento a compresión de la barra biarticulada "imperfecta", figura 3 , se observa que el diagrama $p$-e empieza siendo una linea casi recta y con la misma pendiente que el tramo elástico a tracción. A continuación, a medida que aumenta la compresión, el diagrama $p$-e se va curvando y disminuye progresivamente su pendiente. Esto es una consecuencia directa de las flexiones inducidas por el axil, que acentúan la combadura inicial de la barra aproximando sus extremos en mayor medida de lo que corresponderia al acortamiento en compresión simple. Este comportamiento, aunque no lineal, se mantiene 


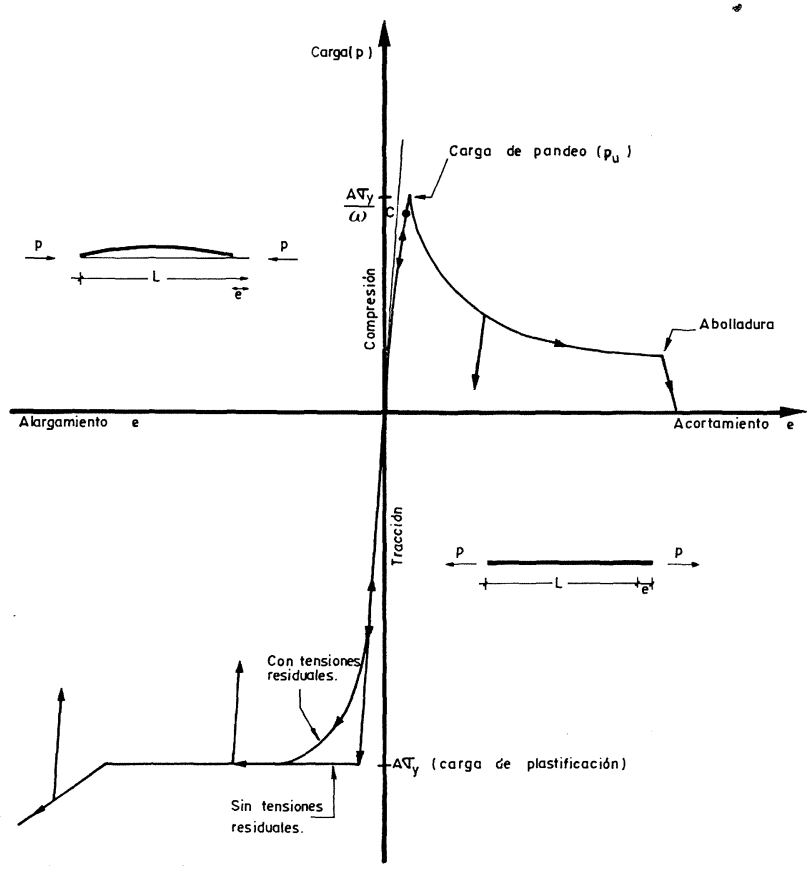

Fig. 3. - Curva constitutiva de una barra biarticulada solicitada por cargas axiles de tracción o compresión.

elástico siempre que la acción combinada del axil y del flector no llegue a producir plastificaciones en la barra. Asi, hasta que no se alcanza esta situación, punto $\mathrm{C}$ del diagrama p-e, el comportamiento de la barra es reversible coincidiendo el camino de carga con el de descarga. Una vez se supera dicho valor, el comportamiento de la barra deja de ser elástico $y$, a medida que se extiende la plastificación por sus secciones más solicitadas, se va acentuando la pérdida de rigidez de la barra. Con ello aumenta su combadura y, en consecuencia, también aumentan las flexiones secundarias inducidas por el axil. Todo esto precipita rápidamente el colapso de la barra, que se produce cuando se alcanza el punto más alto del diagrama p-e. A partir de este momento, si se desea seguir dibujando el diagrama $p$-e para acortamientos "e" crecientes, es necesario disminuir la compresión con el fin de obtener configuraciones deformadas en equilibrio. Por último, la ruina total de la barra suele ir acompañada de un proceso de inestabilidad local (abolladura) en la zona de la superficie de la barra en la que los acortamientos unitarios alcanzan una cierta magnitud tope ligada a su esbeltez geométrica.

El máximo del diagrama $p$-e de la barra comprimida define su carga de pandeo, la cual está firmemente establecido que depende de la magnitud de las «imperfecciones» de la barra.

La postura que adopta la Convención Europea de la Construcción Metálica (C.E.C.M.) sobre las «imperfecciones» de los perfiles huecos redondos, consiste en suponerlas englobadas en dos «imperfecciones tipo», que son:

A) Una combadura inicial de la barra de tipo senoidal y con una amplitud máxima en su sección central de L/1000.

B) Una reducción del $5 \%$ en el limite elástico a compresión del material estructural, siempre que se trate de tubos conformados en frio y terminados por soldadura.

Con la «imperfección tipo A" se pretende considerar el efecto de la combadura real de la barra y la excentricidad en la aplicación de la compresión, mientras que con la «imperfección tipo B" se pretende considerar el efecto de las tensiones residuales inducidas al soldar el tubo. Partiendo de estas «imperfecciones tipo» la C.E.C.M. hace un análisis no lineal de tubos con distintas esbelteces, deduce sus cargas de pandeo y las tabula adimensionalmente en la curva $A_{0}$ de sus «Recomendaciones para la construcción metálica» (1).

Más difíciles de interpretar son los valores de las cargas de pandeo que se obtienen aplicando la norma española MV 103 «Cálculo de las estructuras de acero laminado en la edificación» (2). Recordemos que la tabla de coeficientes $\omega$ contenida en la citada norma está basada en la teoría de la preflecha convencional debida a Dutheil (3). En esta teoria se supone que todas las "imperfecciones» de la barra comprimida se pueden englobar en una «imperfección tipo» consistente en admitir que la barra tiene una combadura latente (no real) de tipo senoidal y con una amplitud máxima de valor

$0,3 \sigma_{y} \mathrm{~W} /\left(\sigma_{\mathrm{E}} \mathrm{A}\right)$. Además, supone que el colapso de la barra comprimida, es decir, el máximo del diagrama $\mathrm{p}-\mathrm{e}$, se produce cuando la tensión en la fibra más solicitada alcanza el límite elástico del acero estructural. Obviamente, las dos premisas en que se basa la teoria de Dutheil son totalmente arbitrarias y se justifican porque conducen a una curva del coeficiente $\omega$ que queda ligeramente del lado de la seguridad con respecto a la nube de puntos correspondientes a los resultados experimentales manejados por este investigador. Sin embargo, plantear sólo una curva de pandeo para todo tipo de secciones transversales, conduce a resultados muy conservadores $\left({ }^{*}\right)$ para los perfiles huecos redondos, puesto que se da la circunstancia de que estos perfiles son los que mejor comportamiento presentan frente a los fenómenos de inestabilidad.

No cabe la menor duda de que con el transcurso del tiempo acabará por imponerse la postura propugnada por la C.E.C.M.,

(*) Excepto en un pequeño tramo de la curva de pandeo corres pondiente a barras cuya esbeltez mecánica es inferior a 50. 
desplazando a formulaciones más antiguas y, consecuentemente, menos elaboradas, como la citada de Dutheil.

\section{MODELO MATEMATICO PARA EL ANALISIS DE BARRAS BIARTICULADAS "IMPERFECTAS" SOLICITADAS A' COMPRESION}

El tercero de los autores de este artículo ha puesto a punto un programa de ordendor con el que se pueden hacer análisis no lineales (mecánicos y geométricos) de entramados metálicos planos. Dicho programa permite considerar el comportamiento elastoplástico del material estructural, la existencia de tensiones residuales en los perfiles, la extensión de la plastificación a lo largo de las barras, los procesos de descarga en las zonas previamente plastificadas y los esfuerzos secundarios provocados por el cambio de geometría inducido en la estructura por la solicitación. El análisis puede llevarse más allá del colapso del sistema estructural con el fin de investigar su comportamiento postagotamiento. Se trata, pues, de una formulación que realiza un análisis muy completo, si bien la complejidad inherente requiere un esfuerzo numérico importante.

En la referencia (4) se puede consultar una exposición detallada de este programa y, por ello, aqui sólo daremos una breve enumeración de los principios en los que se basa.

Limitándonos a considerar el análisis de barras aisladas, estos principios son:

1. Se empieza dividiendo la barra en varios tramos, de forma que ésta pasa a ser considerada como un sistema estructural obtenido por ensamble de los tramos en los que previamente se ha descompuesto. Los puntos de unión de los tramos serán los "nudos" de esta "estructura".

2. Para referir el comportamiento de la estructura se selecciona un sistema de ejes global, ejes $X$ e $Y$ de la figura 4, que se adopta como sistema de referencia en el que se define el vector carga $\bar{F}$ que solicita a la estructura y el vector movimiento $\bar{D}$ de sus nudos. Por otra parte, para referir el comportamiento de cada uno de los tramos se seleccionan dos sistemas de ejes: los ejes locales, ejes $x_{0}$ e $y_{0}$ de la figura 4 , y los ejes intrinsecos, ejes $x$ e y de la misma figura. Los primeros se adoptan como sistema de referencia en el que se define el vector carga $\bar{f}$ aplicado en los extremos del tramo y el vector movimiento đde estos extremos, mientras que el segundo se adopta como sistema de referencia en el que se definen las deformaciones intrinsecas. $\in(x), \chi(x)$ y los esfuerzos intrínsecos $N(x), M(x)$ de cualquiera de las secciones del tramo.

3. Se admite que el análisis de los tramos referidos a sus ejes intrinsecos sólo requiere la consideración de las no linealidades mecánicas, manteniéndose lineales las ecuaciones que relacionan los corrimientos con las deformaciones y los esfuerzos con las cargas.

4. Se admite que todas las no linealidades geométricas se incorporan al análisis al plantear los sucesivos cambios de ejes que permiten pasar de los ejes intrínsecos a los locales y de éstos a los globales.

5. En cuanto a la forma de plantear el análisis, se recurre a un proceso incremental iterativo del tipo Newton-Raphson modificado en la forma expuesta por Riks (5). De este modo se puede seguir todo el diagrama carga-movimiento de la barra comprimida, en nuestro caso la curva p-e, tanto en su rama creciente hasta el pandeo como en la decreciente posterior al mismo.

El modelo de análisis que acabamos de exponer representa una formulación alternativa a otros modelos que también aplican algoritmos incrementales iterativos y que se basan en el método de los elementos finitos (6).

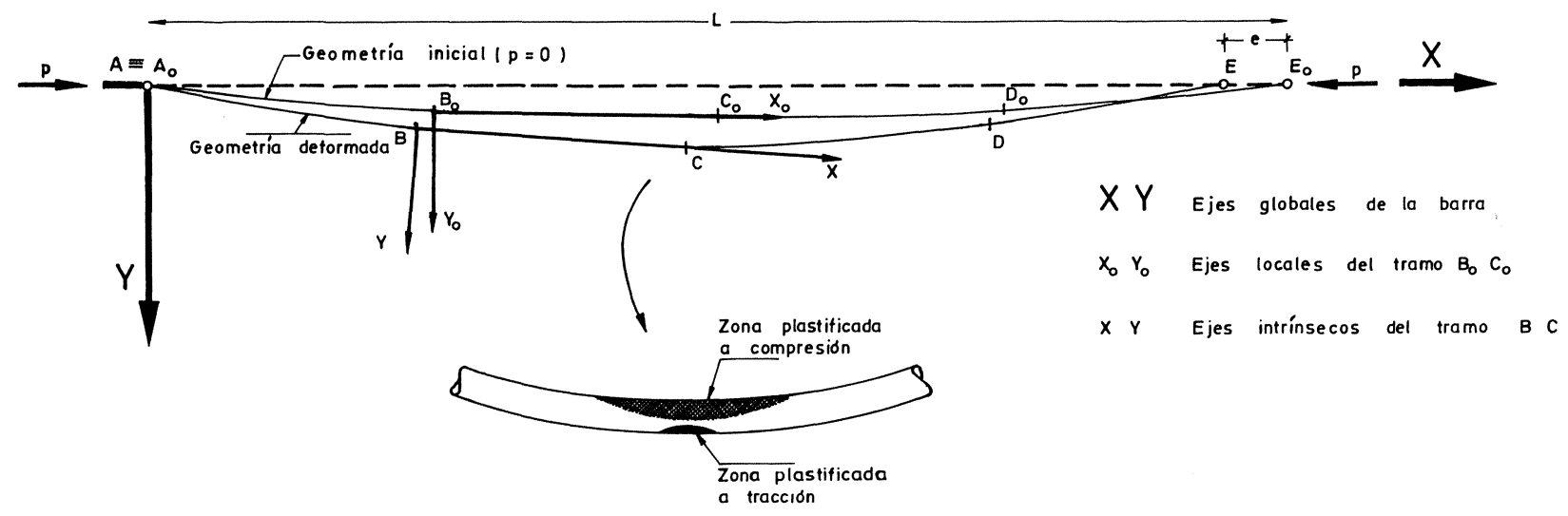

Fig. 4. - Sistemas de ejes utilizados en el cálculo no lineal de barras biarticuladas. 
Formulaciones más simplificadas son las que recurren a la integración directa por métodos numéricos de las ecuaciones de equilibrio de la barra a partir de relaciones esfuerzos generalizados - deformaciones generalizadas de sus rebanadas deducidas previamente (7). Estas formulaciones, aunque tienen en cuenta la extensión de la plastificación a lo largo de la barra, presentan el inconveniente de que no pueden tratar los procesos de descarga en las zonas plastificadas. Finalmente, los modelos de análisis más simplificados son los que suponen a priori la forma deformada de la barra, normalmente una senoide (8). Su ventaja principal es la sencillez y el inconveniente, lógicamente, la falta de precisión, ya que sólo consiguen satisfacer el equilibrio en una sección transversal de la barra, normalmente la sección central.

\section{CURVAS CONSTITUTIVAS DE BARRAS BIARTICULADAS «IMPERFECTAS» SOLICITADAS A COMPRESION}

El programa de ordenador descrito en el apartado anterior nos ha permitido analizar el comportamiento resistente de barras de pared delgada y sección circular, geométricamente imperfectas, articuladas en sus dos extremos y solicitadas por cargas de compresión aplicadas de forma que su línea de acción es la que une los extremos de la barra en su geometria deformada.

En la figura 5 se representan los valores de las cargas de pandeo que hemos obtenido para tubos de acero A-4 $2 b$ de distintas esbelteces. Las curvas punteadas se han deducido suponiendo que todas las «imperfecciones" quedan englobadas en una «imperfección tipo» consistente en una combadura senoidal con una amplitud máxima de L/1000, 2L/1000 y 4L/1000. Admitiendo que las imperfecciones son las propuestas por la C.E.C.M. se obtiene la

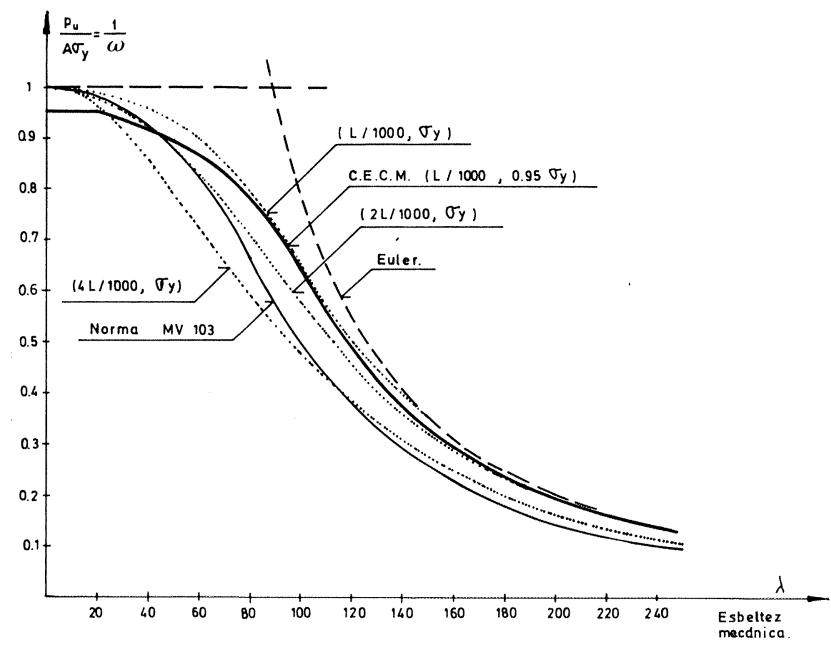

Fig. 5. - Curvas de coeficientes $\omega$ para tubos de acero A-4 $2 b$.

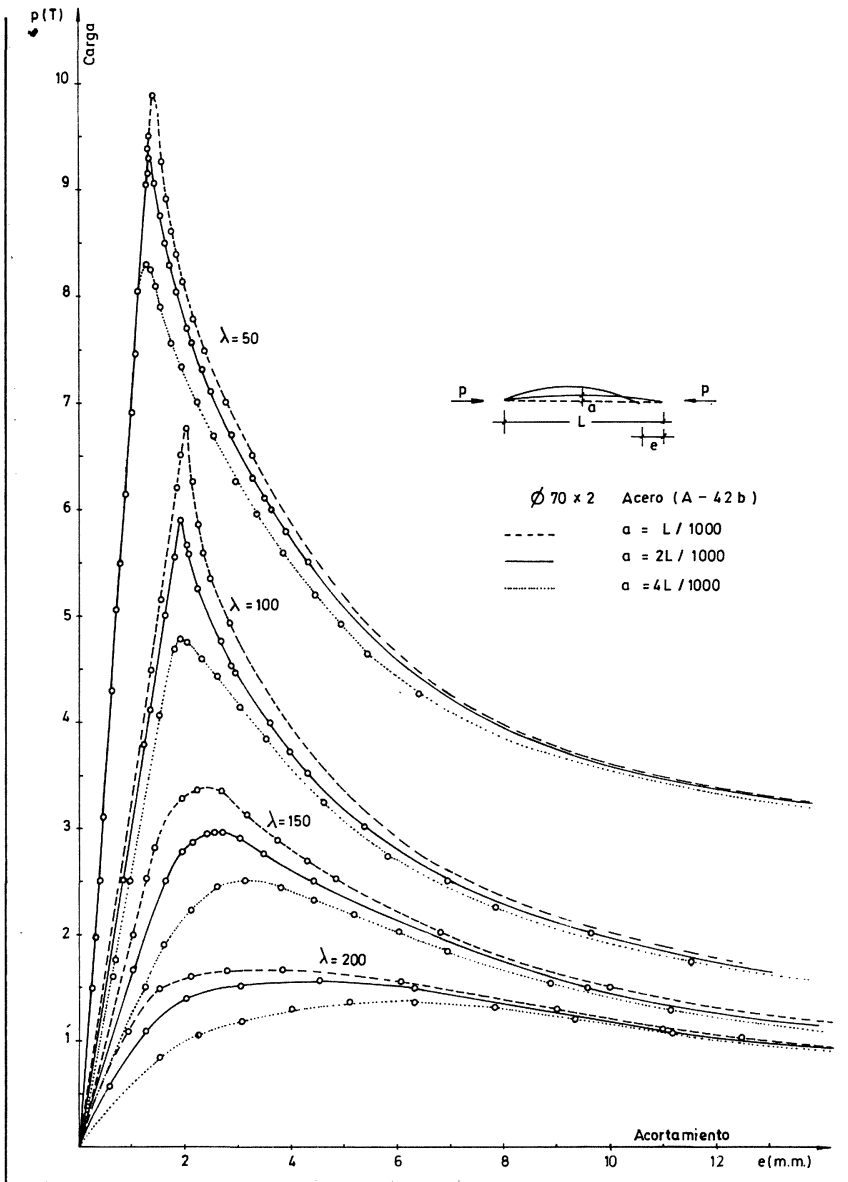

Fig. 6. - Curvas constitutivas de una barra biarticulada comprimida con distintas esbelteces mecánicas e imperfecciones geométricas.

curva continua gruesa, là cual coincide con la curva $A_{0}$ de la referencia (1). En la misma figura se comparan estos valores con los que se obtienen aplicando directamente la norma $\mathrm{MV}-103$, que se han representado mediante una curva continua fina.

En la figura 6 se representan los resultados completos correspondientes al análisis de un perfil $\varnothing 70,2$ de acero $A-42 b$. Se han corisiderado cuatro longitudes diferentes $L=$ $1,20,2,40,3,60$ y $4,80 \mathrm{~m}$, que se corresponden, respectivamente, con unas esbelteces mecánicas de $\lambda=50,100,150$ y 200. Para cada una de las esbelteces consideradas se ha supuesto que la barra presenta una combadura inicial de tipo senoidal con una amplitud máxima en el centro de L/1000, 2L/1000 y 4L/1000. Como hemos indicado, los máximos de los diagramas $p$-e definen las cargas de pandeo de estas barras, las cuales, lógicamente, dependen de su esbeltez y de la imperfección supuesta.

Es evidente que las curvas p-e de la figura 6 sólo son válidas para los ejemplos analizados. Sin embargo, en virtud del teorema $\pi$ de Buckingham-Vaschy (9), si se hacen adimensionales las escalas del dibujo de forma 


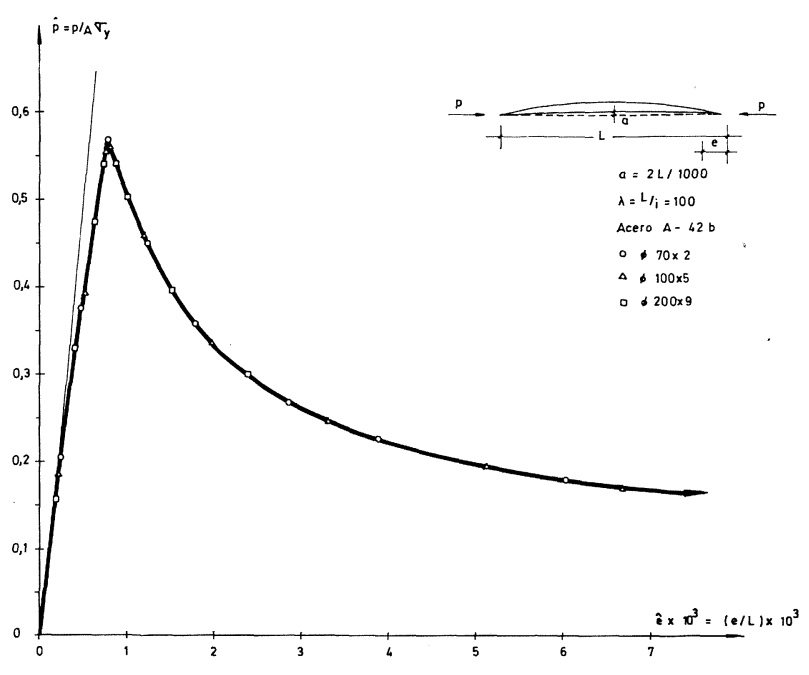

Fig. 7. - Curva constitutiva adimensional de tubos biarticulados comprimidos de esbeltez mecánica $\lambda=100$ e imperfección geométrica $a=$ 2L/1000.

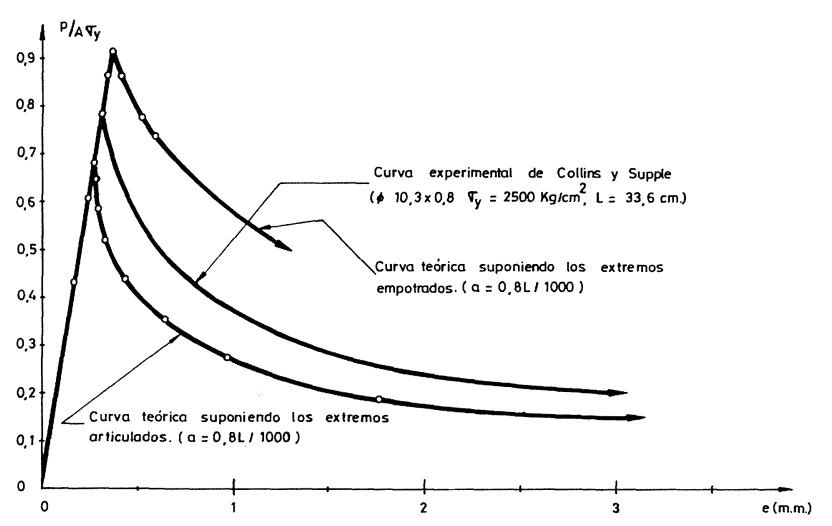

Fig. 8. - Curvas constitutivas adimensionales de tubos biarticulados comprimidos (Acero A-4 2b).

que en el eje de abscisas se representa el desplazamiento relativo ê $=\mathrm{e} / \mathrm{L}$ de los extremos de la barra y en eil eje de ordenadas el axil relativo $\hat{\mathrm{p}}=\mathrm{p} / \mathrm{A} \sigma_{\mathrm{y}}$ que la solicita, se consigue que los diagramas $\hat{p}-\hat{e}$ resultantes pasen a tener validez general para todo tipo de tubos de pared delgada que presenten la misma esbeltez y la misma imperfección geométrica. Esto puede verificarse en la curva adimensional de la figura 7 en la que se superponen con total precisión los resultados correspondientes a tres tubos distintos $(\varnothing 70,2, \varnothing 100,5$ y $\varnothing$ $200,9)$, pero todos ellos de la misma esbeltez mecánica $(\lambda=100)$ y con el mismo nivel de imperfección geométrica (2L/1000).

Por otra parte, conocidas las curvas adimensionales $\hat{p}$-ê correspondientes a dos esbelteces pröximas $\lambda_{1}$ y $\lambda_{2}$, la curva $\hat{p}-\hat{e}$ correspondiente a cualquier otra esbeltez $\lambda$ intermedia pueden obtenerse de forma aproximada por interpolación.
En el caso que nos ocupa estimamos que se obtiene suficiente precisión trabajando con siete curvas adimensionales $\hat{p}$-ê, las correspondientes a las esbelteces mecánicas $\lambda$ $=0,20,50,100,150,200$ y 250 . Estas curvas se definen por puntos al ordenador y aqui se han representado en la figura 8 .

Finalmente, para contrastar la validez del modelo de cálculo aplicado, hemos utilizado los resultados experimentales de Collins y Supple (10). Los ensayos de estos investigadores se realizaron en un equipo de medida computerizado con el que se obtuvieron los diagramas $p$-e de tubos de esbelteces $\lambda=50$, $60,70,80,90,100,110$ y 120 . En todos los casos los tubos estaban aplastados en sus dos extremos y la zona aplastada quedaba aprisionada por las mordazas de la prensa. Esto supone un grado de vinculación del tubo en sus extremos que es una situación intermedia entre las de simple articulación y empotramiento perfecto.

Dado que no se conocen las «imperfecciones" de los tubos ensayados, se han tenido que seleccionar unas «imperfecciones tipo" tales que, en cada caso, la carga de pandeo observada experimentalmente coincida con el valor promedio de los resultados numéricos obtenidos en los supuestos limites de extremos articulados o extremos empotrados. En todos los casos se ha podido verificar que la curva experimental queda situada en la zona comprendida entre las curvas teóricas correspondientes a los dos casos limites supuestos. A modo de ejemplo, en la figura 9 se muestra un resultado tipico correspondiente a un tubo cuyos datos se indican en la misma figura.

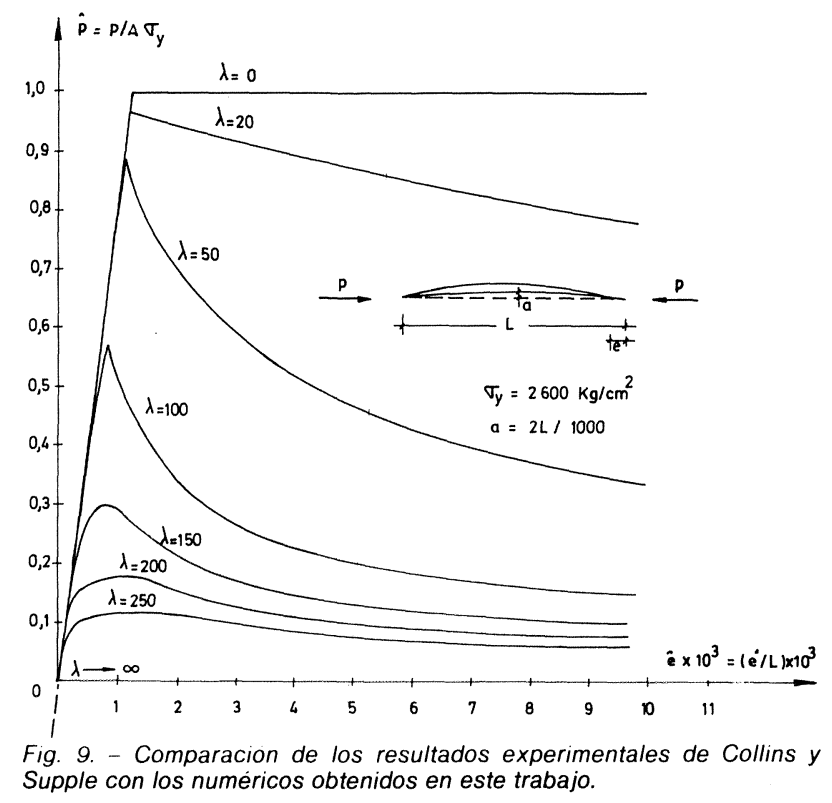




\section{CONCLUSION}

Se ha expuesto un modelo matemático para el análisis no lineal de barras «imperfectas» solicitadas por cargas axiles. Como caso particular se ha estudiado el comportamiento resistente de las barras tubulares de pared delgada que se utilizan en la construcción de mallas espaciales. Esto ha permitido deducir sus relaciones constitutivas, es decir, las curvas adimensionales que relacionan la carga axil que solicita a la barra con el movimiento relativo de sus extremos medido en la dirección del eje que los une en la geometria deformada. La validez del modelo de cálculo utilizado se ha contrastado con resultados experimentales tomados de la bibliografía disponible. Estas relaciones constitutivas serán el punto de partida para el posterior desarrollo de modelos numéricos que permiten estudiar el estado limite último en las mallas espaciales de nudos articulados.

\section{REFERENCIAS}

1. Recomendations for steel constructions. European Convention for Constructional Steelwork, 1976.

2. Norma MV-103. Cálculo de las estructuras de acero laminado en la edificación. Decreto 1353/1973, de 12 de abril (B.O.E.).

3. Instrucción e.m. 62 para estructuras de acero (3.a edición). Instituto Eduardo Torroja de la Construcción y del Cemento, 1969.

4. Irles Más R.: Un modelo numérico para el análisis del colapso en entramados metálicos. Tesis doctoral leida en la E.T.S. de Ing. de C. C. y P. de Valencia, 1985.
5. Riks, E.: An incremental approach to the solution of snapping and buckling problems. Int. J. Solids Struct. 15, 1979.

6. Usama, Madi R. y Lloyd, D.: A finite element model for determining the constitutive relation of a compression member. Proceedings of the third International Conference on Space Structures. University of Surrey, 1984

7. Chen, W. F. y Atsuta, T.: Theory of BeamColumns (Volume 1), McGraw-Hill Book Company, 1976.

8. Paris, P. C.: Limit Desing of Columns, J. Aeronaut. Sci., Jan. 1954.

9. Sedov, L. I.: Similarity and dimensional analysis in mechanics. Academic Press, 1959.

10. Collins, I. M. y Supple, W. J.: Experimental postbuckling curves for tubular struts. Space Structures Research Centre, University of Surrey, 1979.

\section{NOTACION}

$\sigma_{\mathrm{y}} \quad$ Limite elástico del acero.

$\sigma_{\mathrm{E}} \quad$ Tensión crítica de Euler $\left(\pi^{2} \mathrm{E} / \lambda^{2}\right)$

$\lambda$ Esbeltez mecánica de la barra (L/i)

$\omega \quad$ Coeficiente de pandeo

$\in(\mathrm{x}) \quad$ Alargamiento unitario en la sección de abscisa $\mathrm{x}$

$\chi(x)$. Curvatura en la sección de abscisa $x$

a Amplitud de la senoide que representa la imperfección de la barra

e $\quad$ Desplazamiento relativo de los extremos de la barra medido en la dirección del eje que los une en la geometria deformada (ê $=\mathrm{e} / \mathrm{L}$ )

i Radio de giro de la sección transversal de la barra $(\sqrt{1 / A})$

L Longitud de la barra

p Carga axil que solicita a la barra $\left(\hat{p}=p / A \sigma_{y}\right)$

$\mathrm{P}_{4} \quad$ Compresión que provoca el pandeo de la barra $\left(\mathrm{A} \sigma_{\mathrm{y}} / \omega\right)$

A Area de la sección transversal de la barra

E Módulo de Young

I Inercia de la sección transversal de la barra

W Módulo resistente de la sección transversal de la barra

$N(x) \quad$ Esfuerzo axil en la sección de abscisa $x$

$M(x)$ Momento flector en la sección de abscisa $x$ 